\title{
LEISHMANIOSE VISCERAL NO HDT DE ARAGUAÍNA
}

\author{
Visceral leishmaniosis in hdt of araguaína \\ Gilson de Abreu Viza Júnior', Anderson Barbosa Baptista².
}

\section{RESUMO}

A Leishmaniose Visceral (LV) tornou-se uma das doenças mais importantes no Brasil no atual contexto, em razão da sua incidência com caráter de evolução crônica e envolvimento sistêmico que resulta em alta letalidade. Este relato de caso retrata um paciente com febre e queda significativa do estado geral e outros sinais e sintomas cuja identificação do quadro clínico sustentado por exames complementares para LV foi essencial para o estabelecimento de um tratamento adequado.

Palavras-Chave: Leishmaniose Visceral; Anemia; Anfotericina B desoxicolato.

\section{甶 ACESSO LIVRE}

Citação: Viza Júnior $G$ de $A^{1}$, Baptista $A B^{2}$, (2020). Leishmaniose Visceral no HDT de Araguaína. Revista de Patologia do Tocantins., 7(1):. 119-121

Instituição:'Acadêmico de Medicina da Universidade Federal do Tocantins, Tocantins, Brasil; ${ }^{2}$ Professor Doutor do Curso de Medicina da Universidade Federal do Tocantins, Tocantins, Brasil.

Autor correspondente: : Gilson de Abreu Viza Júnior; gilsonviza@uft.edu.br

Editor: Guedes V. R. Medicina, Universidade Federal do Tocantins, Brasil.

Publicado: 09 de junho de 2019.

Direitos Autorais: (c) 2020 Viza Júnior et al. Este é um artigo de acesso aberto que permite o uso, a distribuição e a reprodução sem restrições em qualquer meio, desde que o autor original e a fonte sejam creditados.

Conflito de interesses: os autores declararam que não existem conflitos de interesses.

\begin{abstract}
Visceral Leishmaniasis (LV) has become one of the most important diseases in Brazil in the present context, due to its incidence with a chronic evolution character and systemic involvmente that results in high lethality. This case report portrays a patient with fever and a significant drop in general condition and other signs and symptoms whose clinical identification sustained by complementary examinations for LV was essential for the establishment of an adequate treatment.
\end{abstract}

Keywords: Visceral Leishmaniasis; Anemia; Anfotericina B desoxicolato 
INTRODUÇÃO

A Leishmaniose Visceral (LV) é causada pelo protozoário intracelular do gênero Leishmania e transmitida especialmente pelo flebotomíneo Lutzomyia longipalpis, tornou-se uma das doenças mais importantes no Brasil sendo a região norte e nordeste as que apresentam a maior incidência ${ }^{1}$. Sua importância está relacionada a razão da sua incidência com caráter de evolução crônica e envolvimento sistêmico que resulta em alta letalidade, sobretudo em indivíduos não tratados e em crianças desnutridas por conta da baixa condição socioeconômica. Além disso, é considerada emergente em indivíduos portadores de infecção pelo vírus da imunodeficiência adquirida ${ }^{2}$.

Em 2017, os delegados dos países endêmicos, participantes da Reunião Regional de Leishmanioses, aprovaram o Plano de Ação de Leishmanioses nas Américas 2017-2022, que detalha as metas, indicadores e ações para cumprir com os compromissos da Resolução CD 55 R09 de 2016, com o objetivo de reduzir a morbidade e mortalidade por leishmanioses na região mediante o fortalecimento do diagnóstico, tratamento, reabilitação, prevenção, vigilância e controle ${ }^{3}$.

A Organização Mundial de Saúde preconiza três medidas profiláticas: destruição do vetor, tratamento dos casos humanos e destruição dos reservatórios que são os cães soropositivos, no entanto é necessário que as ações de educação em saúde sejam concretas e efetivas onde o SUS é o responsável pela participação social e descentralização administrativa ${ }^{4}$.

Existe uma nova realidade de contagio da doença no Brasil, antigamente as pessoas mais expostas e consequentemente mais susceptíveis ao contágio eram os moradores das áreas rurais ou pessoas com o estilo de vida mais rural, nos últimos anos a realidade mudou pois houve um maior acometimento de pessoas com estilo extremamente urbanos isso se deve ao deslocamento dos vetores antes de áreas rurais ou matas agora para áreas urbanas, além do incremento de turismo ecológico ${ }^{5}$.

Este relato de caso tem como objetivo mostrar a importância do diagnóstico de LV e inicio precoce do tratamento quando foram excluídos outros diagnósticos.

\section{RELATO DE CASO}

O. P. B., 32 anos, masculino, pardo, natural de São Geraldo do Araguaia - PA e procedente de Araguaína - TO, deu entrada no Hospital de Doenças Tropicais de Araguaína TO dia 18/04/2019. Na ocasião, o paciente apresentava uma história de febre há mais de 30 dias, contínua, vespertina, associada a astenia, mialgia, perda de peso de $5,0 \mathrm{~kg}$ em 30 dias (sic). Ao exame físico apresentava regular estado geral, lúcido e orientado em tempo e espaço, febril $39,2{ }^{\circ} \mathrm{C}$, acianótico, anictérico, hipocorado 3+/4+, hidratado, PA: $90 \times 60$ $\mathrm{mmHg}$, FC 146 bpm, FR $24 \mathrm{irpm}$, peso 61,6 kg, satO2 98\%. O abdome era plano, RHA presentes, tenso, presença de hepatoesplenomegalia, ausência de dor à palpação superficial e profunda.

Ao exame laboratorial da admissão, paciente apresentava pancitopenia: Hemoglobina 5,2 g/dL, Hematócrito 16,9\%, Leucócitos $1.200 / \mathrm{mm}^{3}$, plaquetas $83.000 / \mathrm{mm}^{3}$, TGP $46 \mathrm{U} / \mathrm{L}$, TGO $30 \mathrm{U} / \mathrm{L}$, FA $82 \mathrm{U} / \mathrm{L}$, PTF 7,0 g/dL, Alb 2,7 g/dL, Glob 4,3 $\mathrm{g} / \mathrm{dL}$, BTF $0,5 \mathrm{mg} / \mathrm{dL}, \mathrm{BD} 0,1 \mathrm{mg} / \mathrm{dL}, \mathrm{Bl} 0,4 \mathrm{mg} / \mathrm{dL}, \mathrm{Ur} 28 \mathrm{mg} / \mathrm{dL}$, Cr 1,04 mg/dL, Na $130 \mathrm{mmol} / \mathrm{L}, \mathrm{K}$ 4,0 mmol/L, Glicemia 104 $\mathrm{mg} / \mathrm{dL}$. Sorologias do dia 04/04 apresentou: Toxo IgM soro não reativo (SNR), IgG R, CMV IgM SNR, IgG R, EBV IgM SNR, IgG R, Brucelose IgM SNR, IgG R, Anti-HIV SNR, HBsAg SNR, Anti-HBs SNR, Anti-HCV SNR.

Nos exames de imagem, a tomografia computadorizada de abdome do dia 05/04/2019 observou-se hepatoesplenomegalia e aumento do calibre da veia porta e veia esplênica; em Ultrassonografia de Abdome anterior do dia 28/03/2018 observou-se sinais de esteatose leve e hepatoesplenomegalia.

Paciente foi internado com suspeita de leishmaniose visceral e iniciado tratamento com Anfotericina B desoxicolato. Além disso, paciente recebeu 3 unidades de hemoconcentrado e tratamento sintomatológico.

No primeiro dia de internação, paciente não apresentou febre, evoluiu com melhora do estado geral e dos exames bioquímicos. No dia 22/04/2019 o resultado do exame para LV foi positivo.

\section{DISCUSSÃO}

O Hospital de Doenças Tropicais se localiza no Norte do Estado do Tocantins, região com grande incidência de LV, que possuem características ambientais favoráveis como a baixa elevação e a alta precipitação pluviométrica, o que pode ser explicado pela maior presença do vetor em altitudes mais baixas, e em locais mais úmidos ${ }^{1}$.

A cidade de Araguaína apresentou números alarmantes de LV, principalmente entre 2006 e 2007 e está entre os quatro municípios com maior número de registros do país. Surtos da doença atingiram progressivamente, a partir de 2008, áreas centrais e periurbanas ${ }^{6}$, chamando a atenção do poder público para a efetivação dos processos profiláticos e educação em saúde.

O diagnóstico preciso e rápido é importante, pois a mortalidade pode atingir $90 \%$ dos pacientes. Os sintomas como febre intermitente, alterações esplênicas, hepáticas, renais, pulmonares e no tecido hemacitopoético podem ser indícios da doença ${ }^{7}$. De acordo com a Vigilância Epidemiológica do Ministério da Saúde todo indivíduo com febre e esplenomegalia, proveniente de área com ocorrência de transmissão de LV ou proveniente de área sem ocorrência de transmissão, descartando os diagnósticos diferenciais mais frequentes da região é considerado caso suspeito de LV$^{8}$.

Há várias possibilidades para a realização do diagnóstico, que é baseado no encontro do parasita no tecido da medula 
óssea, baço, fígado ou linfonodos. Testes sorológicos como imunofluorescência indireta, ELISA e técnicas de biologia molecular, reação em cadeia da polimerase, no entanto como há especificidades variáveis e o custo é alto, pode ser adotada a prática clínica, achados clínico-epidemiológicos e exames mais acessíveis como testes imunocromatográficos, comumente conhecidos como teste rápido, que utilizam antígenos recombinantes; pacientes com resposta favorável ao teste terapêutico são realizados e o tratamento iniciado ${ }^{9,10}$. $O$ tratamento escolhido foi a Anfotericina B desoxicolato como está recomendado pela Organização Pan Americana de Saúde. Logo no primeiro dia de internação o paciente já apresentou cura inicial devido a ausência de quadro febril e melhora do estado geral. A duração do tratamento com esta medicação deve ser de 14 dias e estará completado quando houver 6 meses de ausência de recaída, é preciso ficar vigilante às questões de desenvolvimento de resistência às drogas, o consequente aumento do tempo de internação e maior custo de internação ${ }^{10,11,12}$.

Outra opção de tratamento seria antimônio penta-valente $\mathrm{N}$ metil glucamina (Glucantime), que produz efeitos colaterais como a arritmia, artralgias, adinamia, anorexia, dor no local da aplicação (IM) e aumento da diurese, em casos graves e que essa medicação não responda a escolha será da Anfotericina que pode produzir flebite, cefaléia, febre, calafrios, astenia, dores musculares e articulares, vômitos e hipotensão durante a infusão ${ }^{13}$.

O quadro de anemia foi tratado com hemotransfusão. Nos níveis de hemoglobina inferior a $7 \mathrm{~g} / \mathrm{dL}$ existe grande risco de hipóxia tecidual e comprometimento das funções vitais ${ }^{14}$. Dessa maneira, foram feitas quantidades de hemácias suficientes para correção dos sinais/sintomas de hipóxia.

Esse estudo demonstra a importância de um diagnóstico precoce, e faz uma alerta para a necessidade de medidas de controle e políticas de educação em saúde que sejam efetivas para evitar o contágio, principalmente onde há incidência de cães contaminados e o número de notificações é alto. É necessário que pesquisas em busca de uma vacina evoluam como método definitivo para a prevenção.

Faz se necessário que haja maior investigação científica no Estado do Tocantins de casos suspeitos e notificados, condutas adotadas e óbitos, bem como possibilidades de tratamentos alternativos e complementares, inclusive disponíveis na Relação Nacional de Plantas Medicinais de Interesse ao Sistema Único de Saúde (Renisus). Por termos encontrado pouca publicação da Região Norte do Brasil esse relato fica limitado a uma análise sem maiores detalhes comparativa.

\section{CONCLUSÃO}

A conduta de tratamento imediato baseado no exame clínico, complementado pelos achados laboratoriais para suspeita de Leishmaniose Visceral foi adequada e pertinente, proporcionando ao paciente bom prognóstico e menor tempo de internação, demonstrado pela ausência de febre nos dias que se seguiram à internação e que representam uma prova terapêutica positiva. O diagnóstico foi confirmado pelo critério da Organização Pan Americana de Saúde por meio do teste imunocromatográfico positivo.

\section{REFERÊNCIAS BIBLIOGRÁFICAS}

1. Reis, L.P., Balieiro, A.A.S., Fonseca, F.R., Gonçalves, M.J.F. Leishmaniose visceral e sua relação com fatores climáticos e ambientais no Estado do Tocantins, Brasil, 2007 a 2014. Cad. Saúde Pública, 35 (1) 10 Jan 2019.

2. Vilela, M., Afonso, M., Costa, S., Costa, W., \& Rangel, E. (2014). Lutzomyia (Lutzomyia) longipalpis: Fatores associados ao processo de expansão e urbanização da leishmaniose visceral americana. In Conceição-Silva F. \& Alves C. (Eds.), Leishmanioses do continente americano (pp. 183-192). Rio de Janeiro: SciELO Editora FIOCRUZ Disponível em: http://www.jstor.org/stable/10.7476/9788575415689. $\underline{13}$.

3. Organização Pan-Americana da Saúde: Leishmanioses: Informe Epidemiológico nas Américas: Washington: Organização Pan-Americana da Saúde; 2018 [acesso 19 de abril de 2019] Disponível em: www.paho.org/leishmaniasis.

4. Castro, J.M. et al. Conhecimento, Percepções de Indivíduos em Relação à Leishmaniose Visceral Humana Como Novas Ferramentas de Controle. Ensaios Cienc., Cienc. Biol. Agrar. Saúde, v.20, n.2, p. 93-103, 2016.

5. Silva EG, Silva EG, Lava CO, Santana SC. A enfermagem e a sistematização do atendimento ao portador da Leishmaniose Tegumentar Americana. Rev Cient Fac Educ e Meio Ambiente [Internet]. 2018;9 (ed esp): 507-511. doi: https://doi.org/10.31072/rcf.v9iedesp.640.

6. Toledo, C.R.S., et al. Vulnerabilidade à transmissão da leishmaniose visceral humana em área urbana brasileira. Rev. Saúde Publica, 51:49, 2017.

7. Lima, M.B. \& Batista, E.A.R. Epidemiologia da leishmaniose visceral humana em Fortaleza-CE. RBPS; 22 (1) : 16-23, 2009.

8. Brasil. Ministério da Saúde. Secretaria de Vigilância em Saúde. Departamento de Vigilância Epidemiológica. Leishmaniose visceral: recomendações clínicas para redução da letalidade / Ministério da Saúde. Departamento de Vigilância Epidemiológica. Brasília: Ministério da Saúde, 2011 Disponível em: http://portalms.saude.gov.br/images/pdf/2016/deze $\mathrm{mbro} / 14 / \mathrm{lv}$ reducao letalidade web revisado.pdf.

9. Junior, L.G.C., et al. Leishmaniose visceral infantil: relato de caso. Rev. Med. (São Paulo). jul.-set.; 95(3):133-7, 2016.

10. Organización Panamericana de la Salud. Leishmaniasis en las Américas: recomendaciones para el tratamiento. Washington, D.C.: OPS, 2013.

11. Monge-Maillo $B$, López-Vélez R. Therapeutic options for visceral leishmaniasis. Drugs. ;73(17):1863-88, 2013. doi:10.1007/s40265-013-01330.

12. Alvar J, Croft S, Olliaro P. Chemotherapy in the treatment and control of leishmaniasis. Adv Parasitol.;61:223-74,2006. http://dx.doi.org/10.1016/S0065-308X(05)61006-8.

13. Pereira, M.D., Lopes, J.D., Neves, M.G.C. $145 \mathrm{Com}$. Ciências Saúde. 2015; 26(3/4): 145-150RELATO DE CASOLeishmaniose Visceral em criança: um relato de 
caso sobre a recidiva da doença. Com. Ciências Saúde, 26(3/4): 145-150, 2015.

14. Brasil. Ministério da Saúde. Secretaria de Atenção à Saúde. Departamento de Atenção Especializada. Guia para o uso de hemocomponentes / Ministério da Saúde, Secretaria de Atenção à Saúde, Departamento de Atenção Especializada. - Brasília : Editora do Ministério da Saúde, 2010. 